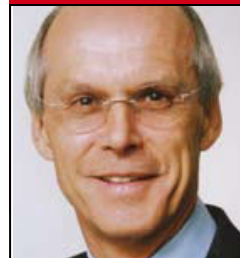

Prof. Dr. med. H. S. FüeßI

Isar-Amper-

Klinikum,

KI. München-

Ost, Haar

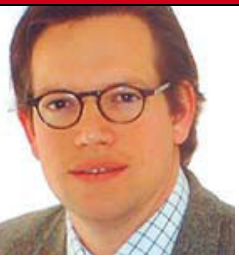

Prof. Dr. med. P. Radke

Universitätsklinikum SchleswigHolstein, Campus Lübeck

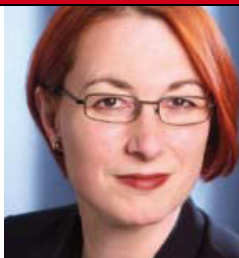

Prof. Dr. med. M. de Zwaan Universitätsklinikum ErlangenNürnberg

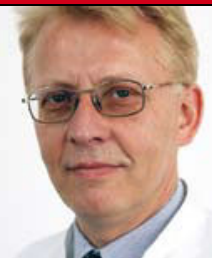

Prof. Dr. med. $K$. Rasche Kliniken St. Antonius, Wuppertal

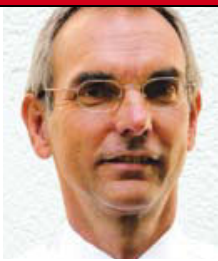

Prof. Dr. med. A. Gillissen Lungen- und Bronchialmedizin, Klinikum Kassel

\title{
PFO-Verschluss bei Migräne nicht zu rechtfertigen
}

\begin{abstract}
Ein möglicher Zusammenhang zwischen einem persistierenden Foramen ovale (PFO) und migräneassoziierten Kopfschmerzen wurde immer wieder postuliert. Eine aktuelle Fallkontrollstudie aus den USA ergab nun, dass die Prävalenz eines PFO bei Patienten mit Migräne ebenso hoch ist wie bei einem beschwerdefreien Kontrollkollektiv. Unter der alleinigen Indikation Migräne sollte ein PFO-Verschluss daher nicht erfolgen.
\end{abstract}

— Etwa 6\% der Männer und 15-18\% der Frauen leiden an Migräne. Beobachtungsstudien an Patienten mit interventionellem Verschluss eines PFO nach kryptogenem Schlaganfall haben einen möglichen Zusammenhang zwischen dem interatrialen Shunt und Migräneanfällen postuliert. Hieraus wurde gefolgert, dass bei einem intrakardialen Rechts-links-Shunt möglicherweise vasoaktive Substanzen oder Mikroembolisationen zur Anfallsauslösung bei Migräne führen können. Es konnte jedoch kein klinischer Vorteil für das interventionelle Vorgehen aufgezeigt werden.

Eine aktuelle US-amerikanische Fallkontrollstudie verglich 144 Migränepatienten mit einer alters- und geschlechts- gleichen Gruppe von Freiwilligen ( $\mathrm{n}=$ 144), die keine Kopfschmerzen beklagten. Das Vorhandensein eines PFO wurde mithilfe der transthorakalen Echokardiografie sowie der transkraniellen Dopplersonografie standardisiert überprüft.

Im Ergebnis zeigte sich kein Unterschied in der PFO-Prävalenz zwischen Migränepatienten und der Kontrollgruppe (26,4 vs. $25,7 \%, \mathrm{p}=0,90$ ) und auch nicht zwischen Migränepatienten mit und ohne Aura (26,8 vs. $26,1 \%$, p $=0,93)$.

\section{Kommentar}

Patienten mit stetig wiederkehrenden Migränekopfschmerzen sind in ihrer Leistungsfähigkeit und Lebensqualität zum Teil deutlich eingeschränkt. Erste Mutmaßungen, dass ein interventioneller PFOVerschluss bei Patienten mit Migräne zu einer deutlichen Reduktion der Beschwerden führen könne, wurden von betroffenen Patienten dankbar aufgenommen. Außerdem wurde häufig der Wunsch nach einem interventionellen PFO-Verschluss bei dieser Indikation geäußert. Die Ergebnisse der MIST-Studie und die fehlende Assoziation der PFO-Prävalenz zur Migräne in der aktuellen Arbeit haben erst

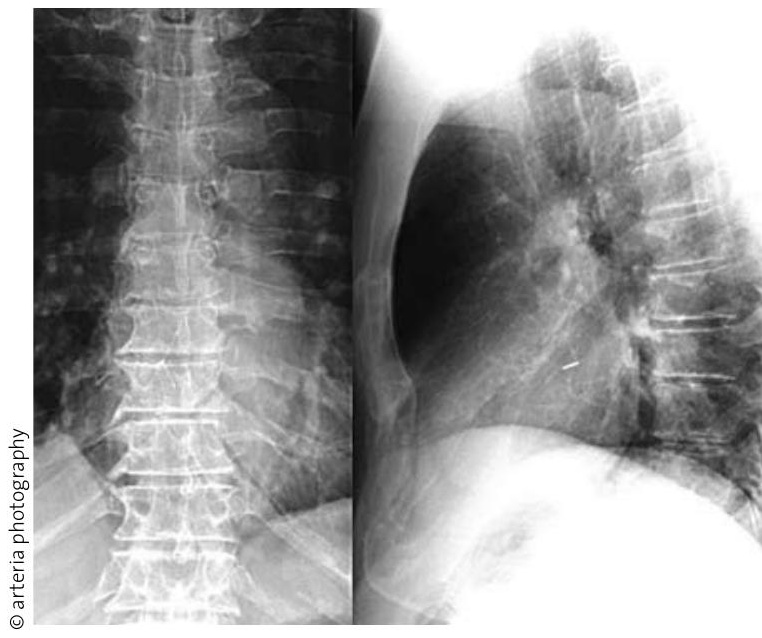

PFO-Verschluss (links in Projektion auf den siebten BWK, rechts im Herzschatten).

einmal eine Menge Wasser in den Wein gegossen. Zum jetzigen Zeitpunkt ist ein interventioneller Verschluss eines PFO mit der Indikation Migräne meines Erachtens nicht zu rechtfertigen. Erst ein tiefer gehendes Verständnis der Triggermechanismen der Migräne kann neue, vielleicht auch interventionelle Therapieoptionen entstehen lassen.

P. RADKE =

\section{- P. Garg et al.}

Lack of association between migraine headache and patent foramen ovale. Results of a case-control study. Circulation 121 (2010) 1406-1412 